\title{
SMC decisions: December 2020
}

In December 2020 decisions, the Scottish Medicines Consortium (SMC) has accepted three new medicines for use by NHS*Scotland.

Venetoclax [Venclyxto] was accepted after consideration through SMC's Patient and Clinician Engagement (PACE) process for the treatment of previously untreated patients with chronic lymphocytic leukaemia (CLL) who have disease-related symptoms. Venetoclax is an oral treatment which can be administered at home and is expected to improve progression-free survival.

Mexiletine [Namuscla] was accepted through the PACE process for the treatment of symptoms of myotonia in patients with non-dystrophic myotonic (NDM) disorders. It is the first medicine to be licensed in Scotland for symptomatic treatment of myotonia in patients with NDM, and may allow some patients to become independent.

Avatrombopag [Doptelet] was accepted through an expedited approach during the COVID-19 pandemic, for the treatment of severe thrombocytopenia in adults with chronic liver disease who are scheduled to undergo an invasive surgical procedure.

However, bempedoic acid [Nilemdo] was not accepted by the SMC for reducing cholesterol levels because the evidence provided by the drug company was not strong enough to satisfy the committee that it would be a good use of NHS resources.

* National Health Service

Scottish Medicines Consortium. December 2020 decisions news release. Internet Document : 7 Dec 2020. Available from: URL: https://www.scottishmedicines.org.uk/aboutus/latest-updates/december-2020-decisions-news-release/ 